\title{
The University of Michigan SMART Center: A Case Study of a Specialized Single Molecule Core Facility's Role in Synergistic Enhancement of Institutional Research
}

\author{
Damon Hoff and Nils Walter \\ University of Michigan, Ann Arbor, Michigan, United States
}

The Single Molecule Analysis in Real-Time (SMART) Center at the University of Michigan (U-M) is a unique shared-use core facility focused on providing access to single molecule tools, including single molecule localization microscopy and particle tracking as well as time-resolved fluorescence microscopy, fluorescence fluctuation spectroscopy, and single molecule force spectroscopy via AFM and optical tweezers, to a broad research community across U-M and regionally. Core facilities perform a vital role in the research community, providing access to specialized tools and expertise to non-specialized labs. Cores also provide an institutional storehouse of technical and practical expertise, allowing both access to a breadth of cutting edge modalities, as well as longevity of information retention. This knowledge base is exploited by users not only for data acquisition, but experimental planning and analysis as well. Our role also extends beyond the activities in the core itself, encouraging relationships between cores on campus as well as between individual labs. As a locus for information and activity involving single molecule techniques, we also foster collaborations across campus when imaging core capabilities are inadequate, connecting investigators with interesting research questions amenable to single molecule techniques with potential collaborators with appropriate tools and expertise to address those questions. These roles synergistically enhance the quality and competitiveness of the institution's research. We will discuss the role of the our core in single molecule research, opportunities and challenges of providing flexible advanced light microscopy resources for a diverse research community, fostering collaborations with other core facilities, and highlight some recent projects undertaken at the SMART Center, including single molecule imaging of RNA turnover in live cells, nanoscale mapping of porous membranes for advanced battery research, and super-resolution probing of gap junction breakdown in the blood-brain barrier after stroke.

\section{References}

Sladojevic N., Stamatovic S.M., Johnson A.M., Choi J., Hu A., Dithmer S., Blasig I.E., Keep R.F., Andjelkovic A.V. (2019) Claudin-1-Dependent Destabilization of the Blood-Brain Barrier in Chronic Stroke. Journal of Neuroscience.

Pitchiaya S., Mourao D.A.M., Jalihal A.P., Xiao L., Jiang X., Chinnaiyan A.M., Schnell S., Walter N.G. (2019) Dynamic Recruitment of Single RNAs to Processing Bodies Depends on RNA Functionality. Molecular Cell 